\title{
TTR
}

Traduction, terminologie, re?daction

\section{Traduire pour l'Afrique. Une approche géo-traducto-logique}

\section{Serigne Kandji, Daouda Ndiaye et Sathya Rao}

Volume 18, numéro 2, 2e semestre 2005

Traduction engagée

Translation and Social Activism

URI : https://id.erudit.org/iderudit/015767ar

DOI : https://doi.org/10.7202/015767ar

Aller au sommaire du numéro

Éditeur(s)

Association canadienne de traductologie

ISSN

0835-8443 (imprimé)

1708-2188 (numérique)

Découvrir la revue

Citer cet article

Kandji, S., Ndiaye, D. \& Rao, S. (2005). Traduire pour l'Afrique. Une approche géo-traducto-logique. TTR, 18(2), 115-133. https://doi.org/10.7202/015767ar

\section{Résumé de l'article}

Ce texte entend s'engager en faveur d'un traduire africain. Pour ce faire, il part du projet de traduction, initié par Daouda Ndiaye, de textes de la diaspora afro-américaine/européenne en langue wolof pour en interroger le geste même : que signifie le fait de restituer à l'Afrique l'héritage de ses fils perdus, ce que le traducteur compte accomplir à travers ses traductions? Est-il possible, par ces traductions en une langue africaine, ici le wolof, de rendre compte de ce qui a été perdu ou retenu durant le terrible voyage, mais aussi de ce qui a été (re)créé sur les nouveaux territoires (la nouveauté des créolisations)?

Mots-clés : Afrique, wolof, Middle Passage, traversée, traduire.
Tous droits réservés @ C TTR: traduction, terminologie, rédaction — Les auteurs, 2007 document est protégé par la loi sur le droit d'auteur. L’utilisation des services d'Érudit (y compris la reproduction) est assujettie à sa politique d'utilisation que vous pouvez consulter en ligne. 


\title{
Traduire pour l'Afrique. Une approche géo-traducto-logique
}

\author{
Serigne Kandji \\ Daouda Ndiaye \\ Sathya Rao
}

\section{Traduire en Afrique}

Le propos de cet article s'inscrit dans le sillage d'un projet de traduction des textes de la diaspora afro-américaine/européenne en langue wolof ${ }^{1}$. Initié par le poète et traducteur sénégalais Daouda Ndiaye, ce projet d'anthologie a été baptisé : Le retour du pigeon voyageur. Celui-ci s'est accompagné d'une œuvre collective de réflexion sur l'acte de traduction dans sa relation, encore insuffisamment explorée, avec la terre d'Afrique en général et le Sénégal en particulier (Rao, 2006a). L’hypothèse, que nous avons explorée au fil de cette réflexion à trois voix, fut la suivante : la question de la traduction, telle qu'elle se pose en Afrique, épouse la complexité de son histoire et de sa géographie. Ainsi, la traduction est-elle un véhicule de premier ordre pour appréhender les déplacements culturels qui y furent (et y sont encore) à l'œuvre. Cette approche globale de la traduction africaine - dont nous serons conduits à discuter les présupposés - sera qualifiée de "géo-traducto-logique ». Nous distinguerons, sans pour autant les opposer, deux ordres « épistémologiques» (Foucault) du traduire africain : un ordre transsaharien et un ordre transatlantique. La raison de cette distinction est essentiellement stratégique : elle permettra d'interroger le double silence qui frappe l'évidence du Dogme et le trauma de la Traite

\footnotetext{
${ }^{1}$ Selon des estimations récentes de la Direction de la Prévision Statistique du Sénégal, près de $90 \%$ de la population du Sénégal comprennent le wolof. Le wolof est également parlé en Gambie et en Mauritanie respectivement par $13 \%$ et $22 \%$ de la population.
} 
transatlantique. Les paradigmes transsaharien et transatlantique ont laissé leurs marques sur le corps culturel de l'Afrique, sous les traits de ce que nous nommerons : la circoncision ${ }^{2}$ et la déchirure ${ }^{3}$. La première signale la rencontre avec l'Islam; la seconde témoigne de la brutalité du contact avec l'Occident colonial judéo-chrétien. Véritables opérateurs dialectiques à l'instar du métissage, de l'hybridité ou de la différance, la circoncision et la déchirure ont toutefois une portée moins universelle, plus régionale et, en définitive, peut-être moins "platonicienne », au sens où pouvait l'entendre Antoine Berman (Berman, 1986). Autrement dit, celles-ci n’ont de légitimité que rapportées à un espace (culturel,

\footnotetext{
${ }^{2}$ Le choix du terme « circoncision » s'accompagne d'une certaine équivoque que nous ne pouvons que reconnaître faute de pouvoir la démêler. Outre la violence même du geste qu'un certain nombre d'organisations internationales ont pris le parti de dénoncer (bien que récemment des études médicales aient démontré les "avantages » d'une telle pratique contre la propagation du SIDA), la circoncision opère la jonction entre des dogmes parfois incompatibles. Si l'usage de la circoncision remonte, à en croire Hérodote, à l'Égypte ancienne, il se retrouve dans les trois monothéismes juif, chrétien (spécifiquement dans les églises coptes d'Égypte et d'Éthiopie) et musulman sous des modalités très différentes. Chose étonnante, la circoncision est également pratiquée dans certaines tribus d'Afrique sans que l'origine en soit clairement établie. En outre, la circoncision, tant décriée par certains (laïques comme chrétiens de tradition paulinienne qui en font l'occasion d'une critique du dogme musulman), est également en vigueur dans nombre de pays anglo-saxons (USA, Canada, Australie), anciennement pour éviter la masturbation conformément à la morale victorienne, aujourd'hui pour des raisons d'hygiène. Et l'on sait à quel point l'hygiène peut avoir partie liée avec l'idéologie en Amérique du Nord. À l'évidence, la circoncision est un opérateur théologico-culturel complexe dont il convient de circonscrire l'usage avec précaution.
}

${ }^{3}$ Faisant directement écho au motif de la circoncision, la déchirure - terme emprunté à Édouard Glissant - témoigne de la violence de la dialectique coloniale. Cette mutilation ontologique exercée sur le corps culturel de l'Afrique peut renvoyer à l' " arrachement » de Frantz Fanon, à la «dissociation » de Jacques Derrida ou bien à l' « écorchure » d'Édouard J. Maunick. Le motif de la déchirure reçoit également des élaborations intéressantes dans les travaux de la psychanalyste et traductrice Janine Altounian. Le propre de ce que nous appelons un matérialisme de la culture consiste à suivre en la décodant la topologie complexe des blessures laissées par l'Histoire sur les corps culturels. Notre propos est ici moins de nous laisser attendrir par excès de sensiblerie (il nous suffit simplement d'en prendre symboliquement acte au plus près du corps) que d'en examiner le parcours, mieux, l'incise, c'est-à-dire le chemin. 
linguistique, géographique, historique) aux propriétés bien spécifiques ${ }^{4}$. En outre, circoncision et déchirure ont ceci de singulier qu'elles portent en elles la violence paradoxale d'une coupure qui pointe vers des destinées communes : celles de l'Oumma (c'est-à-dire la communauté des croyants) et de l'Histoire (c'est-à-dire la communauté des vivants en mémoire, des «remémorés » pour ainsi dire). Plus exactement, c’est dans la performance souvent douloureuse de la « cicatrisation » que se reconstitue et se renouvelle même le lien d'appartenance. Qu'on le veuille ou non, René Girard en aura donné la preuve magistrale, violence et sacré sont presque indissociables. Cette oscillation (entre le pluriel et le singulier, l'universel et le particulier, le sacré et la violence que subit le bouc émissaire) est également inscrite au cœur de la traduction. Bien entendu, les paradigmes de la circoncision et de la déchirure ne sont pas exclusifs; sans compter qu'ils doivent être envisagés dans le détail de leur topologie ${ }^{5}$. Si la conscience de l'ordre transsaharien a été partiellement effacée par l'œuvre d'ensablement du temps (il ne reste en effet que peu de traces historiques de la rencontre entre l'Afrique et l'Islam) et l'évidence du dogme (peu d'Africains questionnent leur héritage musulman); celle, mieux connue, de l'ordre transatlantique demeure encore excessivement visible et douloureuse.

À ces deux espaces du traduire, il faudrait en ajouter un troisième, celui de la traduction immanente à l'Afrique, relevant à la fois de l'ordre précolonial, avec ses supports verbaux et symboliques, et de la grande diversité linguistique de ce continent. Comme le souligne Charles A. Nama (Nama, 1993, p. 414), l’Afrique est une «Babel de langues » (Babel of tongues) qui n'a cessé et ne cesse de pratiquer la traduction au quotidien : on ne recense aujourd'hui pas moins de 2000 langues et dialectes sur le continent africain! Ce troisième paradigme,

${ }^{4}$ Il nous semble qu'un des enjeux fondamentaux des études culturelles aujourd'hui est de parvenir à ébranler l'institution, pour ne pas dire le lieu commun du déplacement dont la globalisation est la figure la plus massivement commerciale. Mettre en mouvement le déplacement (et son avatar qu'est la globalisation), c'est cesser de le considérer de façon monolithique pour mieux percevoir la complexité de ses figures, le comment ça bouge et ses rythmiques.

${ }^{5}$ Dans un texte intitulé « Cultural Identity and Diaspora », Stuart Hall déploie une analytique culturelle similaire, à la différence près que celle-ci opère une coupure supplémentaire - dont nous reconnaissons du reste toute la légitimité (au regard de la singularité des positions d'énonciation) - entre les « présences » américaine et européenne. 
qui est en fait le premier par ordre chronologique, est peut-être celui de la rencontre entre l'Égypte et l'Afrique telle que l'a décrite un Cheikh. A. Diop dans son controversé Nations nègres et cultures ${ }^{6}$ ou, plus récemment, un Théophile Obenga. D’une façon générale, la question de la traduction immanente à l'Afrique n'est pas sans poser un certain nombre de problèmes qui tiennent autant de l'épistémologie que de l'idéologie : 1) comment distinguer les langues africaines entre elles sans tomber dans les écueils successifs de la linguistique coloniale (ou, à l'inverse, afrocentriste), de l'extrapolation à laquelle oblige l'absence de données empiriques ${ }^{7}$ et des luttes régionales pour l'hégémonie linguistique?; 2) comment réaménager le concept de traduction afin qu’il soit délesté de son surplus ethnocentriste (Gyasi, 1999, p. 80) et puisse également valoir, sans préjugés aucuns, pour l'oralité (Nama, 1993, p. 415$)^{8}$. Dans le cadre restreint de cet article, nous nous contenterons d'explorer les modalités géo-traducto-logiques du traduire africain dans les contextes transsaharien et surtout transatlantique où prend place le projet de D. Ndiaye.

L'engagement pris ici se veut d'autant plus rigoureux qu'il tente d'évacuer toute forme d'idéalisme au profit de la théorie entendue comme activité de la pensée en un sens proche de celui d'Althusser. En effet, il nous semble qu'il n'y a pas d'engagement ou d'activisme qui tienne s'il s'arrête au présent de ses propres actes ou revendications, et manque par-là même de se constituer en utopie ${ }^{9}$. L'utopie, que nous

\footnotetext{
${ }^{6}$ Plus exactement, nous faisons référence ici à la thèse afrocentriste de la dérivation des langues africaines (et du wolof en particulier) depuis la langue-mère égyptienne.
}

${ }^{7}$ Le mode de classification génétique - qui fait l'hypothèse de plusieurs proto-langues - favorisé par certains linguistes est loin de faire l'unanimité (Dalby, 1970).

${ }^{8}$ La solution proposée par C. A. Nama, qui s’inspire à la fois des travaux de G. Steiner et de J.C. Catford, consiste à envisager la traduction comme un processus intersémiotique portant indifféremment sur des mots, des sons et des symboles (à l’instar des hiéroglyphes égyptiens, par exemple).

9 À l'heure actuelle, la traductologie assiste à un retour conjoncturel de l'activisme polarisé autour des enjeux du conflit israélo-palestinien (M. Baker), de la subjectivité du traducteur (M. Tymoczko), de la traduction des textes politiques (C. Schäffner), de la lutte postcoloniale (S. Bassnett) et de l'hégémonie de l'anglo-américain (L. Venuti). Or, il nous semble que l'injonction, plus ou moins légitime, à l'action (que ce soit celle des activistes ou 
projetons avec la rigueur d'une théorie (celle d'un matérialisme de la culture à élaborer), consiste à faire entrer comme il se doit l'Afrique dans les champs de la théorie en général et de la traductologie en particulier. Notre propos ici est moins de déplorer le manque d'intérêt des sciences humaines pour l'Afrique que de dénoncer, à la suite des travaux de V. Mudimbe, les reconstructions hallucinantes dont elle a fait l'objet (Rao, 2005). Plus exactement, cet engagement recouvre trois dimensions spécifiques : 1 ) envisager la traduction comme un opérateur susceptible de produire une connaissance de l'Afrique (du type histoire de la traduction en Afrique, modèles traductologiques pour l’Afrique); 2) prendre acte de la spécificité du territoire africain et notamment des marques qu'y a laissées l'histoire en évitant toute pulsion ethno/afrocentrique déformante (attendrissement excessif, mauvaise conscience, victimologie, désir de revanche); 3) simplement traduire en une langue mineure, ici le wolof, et reconnaître - en l'éprouvant par la pratique - la richesse de ses moyens.

\section{Digressions transsahariennes}

Contrairement à ce que nombre d'historiens ont pu dire, le désert du Sahara n'a en rien empêché la rencontre entre l'Afrique et le monde islamique. Il a plutôt donné à cette rencontre une configuration et une topographie singulières. Par un curieux mirage épistémologique, cette topographie n'est pas sans évoquer celle de l'océan qui constitue, nous le verrons, le milieu privilégié de l'ordre transatlantique de la traduction. Ainsi, aux yeux de l'historien Joseph Cuoq, le désert n'est pas une muraille le séparant du reste du monde, mais plutôt une mer intérieure invitant aux traversées. Avec pour rivage le Sahel soudanais (Sahl veut dire rivage en $\operatorname{arabe}^{10}$ ) et la frange sud de l'Afrique septentrionale, le désert saharien constitue un véritable espace de traduction. Depuis presque deux mille ans, celui-ci a accueilli des échanges de nature très diverses entre les mondes islamique et sub-saharien. C'est cette textualité ensablée du désert, dont il demeure encore des traces écrites (récits de marchands arabes comme celui de l'érudit Ibn Battuta) et

des pragmaticiens), a eu pour effet épistémologique d' « inhiber » le travail d’imagination de la Théorie (Rao, 2006b). Au sortir du tournant éthique de la traduction et dans le sillage de la fin des Grands récits, le regain d'activisme pose au fond la question d'un (nouveau) projet politique - peut-être plus humain et local - pour la traduction.

${ }^{10}$ Rappelons que jusqu'au cinquième siècle avant notre ère, le Sahara était un territoire riche, abritant fleuves et lacs. 
physiques (vestiges archéologiques), qu'il faudrait exhumer dans le cadre d'une géo-traducto-logie de l'Afrique.

Facilités par l'introduction du dromadaire, ces échanges ont d'abord été commerciaux. Filant la métaphore maritime, d'aucuns ont pu comparer les caravanes de marchands qui parcouraient les grandes villes du Sahel (Tombouctou, Gao) à des caravelles. L'or, le sel, l'ivoire et les esclaves furent les valeurs d'échange de cette économie du traduire pour le moins dynamique. Il n’y a qu’à lire le récit des premières négociations entre marchands arabo-berbères et noirs pour comprendre à quel point elles tenaient d'un délicat exercice de traduction (entre or, esclaves et marchandises). Il est aujourd'hui admis que c'est le long de ces voies commerciales ou bien à leurs abords que l'Islam a pu commencer à se diffuser en Afrique sub-saharienne. Sans surprise, ce furent les groupes de marchands tels que les Dyoula, les Haoussa et les Dyabhanke qui, les premiers, se convertirent pour devenir les plus fervents promoteurs de l'Islam. Ce n'est que dans un second temps que le travail de conversion en tant que tel fut pris en charge par des clercs et des prédicateurs. Plus généralement, il va sans dire que le processus d'islamisation a pris des formes extrêmement diversifiées, tantôt violentes tantôt pacifiques, selon les époques et les contextes.

La question de la traduction reçoit dans le contexte coranique un traitement tout à fait particulier qui mériterait que l'on s'y arrête. Sans entrer ici dans les détails, nous soulignerons simplement le fait que, si cette question, en contexte islamique, revient, en dernière analyse, à celle de l'intraduisibilité (I'jâz) du Coran, elle trouve, en l'espèce, à se décliner de diverses manières. En effet, cette question de la traduction est étroitement liée à plusieurs thématiques comme : la transmission du Coran, sa mémorisation dans le « cœur » des fidèles, la plasticité de la langue coranique qui continue de «trembler » du fait des divergences articulatoires d'origine dialectale, le caractère hybride de cette langue ou bien encore les modalités de l'exégèse du Coran.

À l'instar du latin en Europe, l'alphabet arabe a permis la retranscription de nombreuses langues africaines (wolof, haoussa, pulaar), donnant ainsi une importance centrale à la question de la traduction (en particulier celle des toponymes). Sans compter que des langues comme le wolof en Afrique de l'ouest et le kiswahili en Afrique de l'est intègrent, dans leur syntaxe et dans leur sémantique, des éléments de l'arabe. Jadis traité de "bâtard " par l'ethnolinguistique coloniale, le kiswahili revendique désormais son "identité multiple » 
(kabila) et métisse (Mazrui et Shariff, 1994). Aussi, n’est-il pas étonnant que certaines formes littéraires swahilies, à l'instar du Utendi, long poème à quatre strophes comportant des rimes finales, s'alignent sur les modèles poétiques arabes. Fort abondante entre le $\mathrm{VIII}^{\mathrm{e}}$ siècle et le XIII ${ }^{\mathrm{e}}$ siècle, la littérature généalogique témoigne de la volonté des groupes africains islamisés d'inscrire l'histoire de leur dynastie dans la continuité de celles du Moyen Orient "blanc » (qu'il soit arabe ou bien perse). Ainsi, l'épopée d'Askia Mohammed et surtout celle de Sundiata frappent-elles en raison de leur syncrétisme, combinant éléments de la culture islamique (monothéisme, patriarcat) et traditions malinké (déités animales, matriarcat). Dans un registre plus actuel, la littérature africaine contemporaine - pensons aux romans de Amadou Kourouma (Le Soleil des indépendances, Monnè outrages et défis) - est riche d'un intertexte arabe et islamique souvent inexploré dans le cadre des études francophones. L'Islam couleur locale peut y apparaître tantôt comme une force d'élévation positive (L'étrange destin de Wangrin de Amadou H. Bâ) tantôt comme un élément contraignant (Vehi Ciosane de Ousmane Sembène) ou bien encore comme les deux à la fois (L'Aventure ambiguë de Cheikh $\mathrm{H}$. Kane). Qui plus est, n'oublions pas qu'un grand nombre d'auteurs africains contemporains de renom (citons, entre autres, A. Hampâté Bâ, C. H. Kane, Birago Diop), bien qu'ils soient francophones, furent d'abord élevés dans la tradition coranique.

Bien qu'elle commence à attirer l'attention des chercheurs occidentaux, l'histoire métisse de la rencontre entre l'Afrique et l'Islam reste encore assez méconnue. Peut-être y a-t-il deux raisons principales à cela. Premièrement, ayant laissé, somme toute, peu de traces, l'histoire transsaharienne de l'Afrique a été mise au second plan du fait de l'ampleur et de la proximité du drame de la traite transatlantique. Tantôt confondue avec cette dernière sous le label unique de colonisation, comme c'est le cas dans l'histoire de la traduction en Afrique qu'esquisse C.A. Nama (Nama, 1993, p. 417) ${ }^{11}$ tantôt tout bonnement oubliée, voire refoulée du fait de sa connivence étroite avec la sphère du théologique ${ }^{12}$, l'ordre transsaharien (de la traduction) doit pouvoir être saisi dans sa spécificité. Dans cette mesure, la métaphore océanique

\footnotetext{
${ }^{11}$ Peut-être faut-il voir dans ce choix, plus ou moins conscient, de valoriser, pour ne pas dire d'hypostasier, une certaine vision de l'Afrique et du statut du traducteur ? Du point de vue de notre géo-traducto-logie de l'Afrique, il n’y pas lieu d'établir de hiérarchies entre les trois paradigmes africains du traduire.

${ }^{12}$ Connivence qui suscite soit le silence dogmatique des fidèles soit la peur de la profanation de la part des non-croyants.
} 
risquerait d'occulter la circoncision - c'est là précisément son mirage autant qu'elle permet de l'appréhender. Deuxièmement, d'un point de vue épistémologique cette fois, l'Afrique se donne généralement à lire et à comprendre à travers un corpus de textes gréco-occidentaux. Ceux-ci constituent, selon le mot de Valentin Mudimbé, l'essentiel de la «bibliothèque coloniale ». Or, comme le souligne à juste titre Ousmane Kane dans une publication récente (2003), le temps est venu d'ajouter à cette bibliothèque « europhone » son pan arabophone afin d'avoir une connaissance plus complète de l'Afrique. C'est précisément ce à quoi s'attachent certains chercheurs qui travaillent, depuis plus de trois décennies, à la constitution d'une telle bibliothèque riche de milliers de textes, traduits et non traduits, abordant des sujets aussi divers que la grammaire, les mathématiques, le soufisme ou le droit. Ces textes proviennent de sources arabes et africaines (principalement mauritaniennes, maliennes et soudanaises). Cela dit, il ne faudrait pas pour autant idéaliser les termes de la rencontre entre l'Afrique et l'Islam comme le font certains historiens, qui passent sous silence la réalité de la traite négrière transsaharienne ${ }^{13}$.

\section{Traduire (dans) ${ }^{14}$ le Middle Passage}

On ne parle jamais qu'une seule langue.

On ne parle jamais une seule langue.

Jacques Derrida

Cet espace complexe de traduction issu de la rencontre entre l'Afrique et l'Islam, en côtoie un autre, à la fois plus familier et plus tragique, celui de l'océan atlantique scène du drame de la traite transatlantique. Loin d'être indifférents l'un à l'autre, ces espaces de sens se touchent et ce, pas seulement métaphoriquement ${ }^{15}$. En témoigne, par exemple, l'étrange

${ }^{13}$ Bien que s’étant étalée sur huit siècles, la traite négrière transsaharienne n’a rien à voir quantitativement (ni même qualitativement d'ailleurs puisqu'il s'agissait surtout d'un esclavage « de service » principalement féminin) avec celle, aux dimensions industrielles, à laquelle se livrèrent les pays occidentaux.

14 Loin d'être un ornement postmoderne, les parenthèses signifient ici la coïncidence entre l'acte de traduction et le mouvement triangulaire de la Traite transatlantique. Autrement dit, traduire le Middle Passage, c’est nécessairement parcourir (dans la langue) la trajectoire ou l'espace qu'il met en scène.

${ }^{15}$ Dans cette optique, un ouvrage récent d’Olivier Pétré-Grenouilleau intitulé Les traites négrières s'efforce, pour la première fois, de donner une vision du phénomène de la Traite négrière dans sa globalité. 
destin des marabouts en Afrique de l'est, qui ont su tirer avantage de leur pouvoir symbolique pour s' "accommoder" politiquement et économiquement de la présence des colons européens (Robinson, 2000). Dans le même ordre d'idées, on mentionnera le paradoxe historique qui veut qu'en quelques décennies, nouvelles infrastructures et violentes répressions aidant, la colonisation européenne ait permis beaucoup plus de conversions à l’Islam que neuf siècles de prosélytisme!

C'est à l'intérieur de ce cadre spatio-temporel - qui s'étend grosso modo du $\mathrm{XVIII}^{\mathrm{e}}$ au milieu du $\mathrm{XIX}^{\mathrm{e}}$ siècle et met en relation des territoires aussi éloignés que l'Amérique, l'Europe, l'Afrique et les Caraïbes - que nous souhaitons camper notre réflexion sur l'acte de traduction. Comme le rappelle D. Ndiaye, un des enjeux de la traduction de textes de la diaspora afro-américaine/européenne en langue wolof n'est autre que la possibilité même du retour d' " africains arrachés à leurs terres et réduits en esclaves en Amérique du Nord, dans les Caraïbes et en Amérique du Sud » (citation tirée de: Le retour du pigeon voyageur, à paraître). Possibilité que pourrait bien compromettre le processus d'acculturation forcée auquel ces esclaves ont été soumis et le silence relatif (voire la méconnaissance souvent organisée) entourant la tragédie de la traite transatlantique du côté africain. Aussi, traduire (dans) le Middle Passage, selon l'expression de Paul Gilroy (1992), engage, nous semble-t-il, à un double geste, fondateur d'une traductologie inédite : refaire en sens inverse le terrible chemin des négriers et, ce faisant, restituer à l'Afrique ce qu'on lui a arraché. En d'autres termes : rappeler à la vie une langue africaine - le wolof - dont le maître voulait se lester en s'efforçant de ne pas perdre, au passage, la mémoire des déportés, épelée dans une langue non africaine. En définitive, traduire (dans) le Middle Passage, c'est revenir à la langue d'origine en tentant de témoigner dans une langue africaine - c'est là que se situe précisément l'œuvre de création - du terrible chemin qui a été accompli. Ce nouveau parcours géo-traducto-logique - qui est probablement le plus difficile puisqu'il porte l'épreuve du retour à l' " origine » réelle et non plus fantasmée - prolonge l'itinéraire romantique de la traduction afro-américaine de l'Afrique (pensons aux chantres de la Renaissance de Harlem) et celui, plus contemporain, des écritures postcoloniales. Ici, nous nous référons plus spécifiquement aux phénomènes complexes d'interférences entre langues africaines et langues coloniales (anglais, français) à l'œuvre chez certains auteurs comme Nazi Boni, Ngugi wa Thiong’o ou Gabriel Okara. Procédant souvent d'une volonté de 
résistance ou bien d'un désir d'adaptation plus ou moins conscient, ces tentatives d' " africanisation » ont pu être mises sur le compte d'une performance de traduction (Bandia, 2001). Sur un autre registre, la perspective du retour force également à reposer la question de la créolisation depuis la terre d'Afrique ${ }^{16}$, depuis cette "présence " africaine selon le mot de Stuart Hall.

Si l'ordre transsaharien se rappelle à la mémoire du poète-traducteur par l'expérience cicatrisée de la circoncision, l'ordre transatlantique l'interpelle, quant à lui, par les lacérations de la béante déchirure. La topologie des paisibles étendues de sable s'offre comme lieu de conversion, de conversation et de conservation, et les langues africaines en ressortent avec une identité renouvelée (à l'image du wolofal écrit par les marabouts, lequel combine éléments linguistiques du wolof et de l'arabe). Par contraste, la topologie des terribles immensités océanes s'entend comme ce lieu où toute langue d'origine est tu(é)e, où tout don constitutif antérieur ne résiste que parce qu'il est intériorisé, pour ne pas dire refoulé :

When we were first brought here from our innumerable African tribes, each of us spoke the language of his tribe. But the Lords of the Land decreed that we must be distributed upon the plantations so that no two of us who spoke a common tongue would be thrown together, lest we plot rebellion. So they shackled one slave to another slave of an alien tribe. Our eyes would look wistfully into the face of a fellow-victim of slavery, but we could say no word to him. Though we could hear, we were deaf; though we could speak, we were dumb! (Wright, [1941] 1997, p. 169).[Quand nous fûmes amenés ici pour la première fois en provenance de nos innombrables tribus africaines, chacun de nous parlait la langue de sa tribu. Mais les Seigneurs de la Terre décrétèrent que nous devions être dispersés entre les plantations de façon à ce que pas un d'entre nous ne se retrouve avec quelqu'un parlant la même langue au cas où nous fomenterions un complot. Aussi, prirent-ils soin d'enchaîner ensemble des esclaves de tribus différentes. Nos yeux examinaient, avec nostalgie, le visage de notre compagnon d'infortune sans que nous ne puissions lui souffler mot. Quand bien même nous entendions, nous étions sourds, quand bien même nous parlions, nous étions muets!] (notre traduction)

\footnotetext{
${ }^{16}$ Certains créoles, comme ceux parlés au Surinam ou au Brésil, conservent encore l'héritage syntaxique des langues africaines, respectivement le kwa et le yoruba ou le kongo (Heine/Nurse, 2000, pp. 9-10).
} 
La langue du maître qui a traversé le désert trouvait son assise dans les cœurs et la mémoire des convertis (Coran), la langue du maître qui a fait traverser l'océan s'est attaquée à la mémoire de ses hôtes et s'est dressée contre le chœur des convicts. Pour autant, il n'y a pas lieu de trancher entre l'éternelle évidence de l'Islam (tenue trop facilement pour acquise) et la coupure traumatique de la Traite transatlantique (qui ne semble jamais pouvoir cicatriser). Il s'agit tout simplement de concevoir l'acte de traduction qu'entreprend Le retour du pigeon voyageur comme alliant transfert de sens et de mémoire, comme allant de l'un à l'autre, comme retour chargé.

«Tekki (ci) digguw yoon» est la traduction que propose D. Ndiaye pour "Traduire (dans) le Middle Passage », en soulignant d'abord la polysémie induite par le mot tekki. Il retient trois sens: " dénouer » (herméneutique), " traduire » (linguistique) et "réussir » (pragmatique). Ce qui signifie que dans son travail de traduction, il est confronté d'emblée à un nœud : l'africanité de ces Africains que temps et espace séparent de l'Afrique ${ }^{17}$. Il lui faut donc à la fois dénouer ce jeu et déjouer ce nœud. Dans un premier mouvement, il lui appartient non seulement de traduire en assumant que sa propre langue (le wolof en l'occurrence) eut pu être la langue coupée sur l'autre rive, c'est-à-dire en s'assurant qu'elle est totalement autre, car cette langue a, bien entendu, sa propre histoire. Dans un second mouvement, il s'agit de traduire en se rappelant que les textes qu'il a choisis — de par leur appel présumé ou avéré à l'Afrique — ont surtout (re)construit quelque chose d'inaliénable et même à certains égards d'intraduisible. Il s'agit au final de réussir à res(t)ituer à l'Afrique, par le truchement d'une langue africaine, le chant de ses fils/filles du lointain sans pour autant sous-tendre que la diaspora noire vit constamment la nostalgie d'une patrie perdue, mais au contraire sous-entendre que si motivation nostalgique il y a, c'est-à-dire, regret de ce qu'on n'a pas connu, c'est plutôt du côté de l'Afrique qu'il faudrait se tourner, car c'est elle qui ne connaît pas le chant de ses fils/filles perdus ou qui ne le connaît principalement qu'à travers des langues qui lui sont étrangères.

Notre assise géo-traducto-logique n'est autre que ce Middle Passage, espace à la fois fixe et mobile, mais espace dont le contour, le contenu restent indéterminés : combien d'âmes perdues? Et sous quel parallèle? Seule la langue peut traduire ici, mais la langue retrouvée

\footnotetext{
${ }^{17}$ « What's Africa to me ? » s'interroge Countee Cullen dans un poème intitulé « Heritage ».
} 
risque aussi de trahir. Or, la perte assumée d’une partie de l'héritage n'est-elle pas la meilleure façon d'exorciser toute nostalgie mal placée (particulièrement celle à l'endroit de la langue-source)? Si la traversée, aussi terrible fût-elle, ne s'est pas faite sans pertes, elle doit s'accompagner d'un nécessaire devoir de mémoire : rendre hommage à ceux - hommes et mots - qui n'ont pas survécu aux voyages (ceux de la traversée-traduction). C'est cela même que le mot wolof digg dans digguw yoon veut traduire. Cet « entre-deux », qui est aussi la traversée que le poète-traducteur accomplit inlassablement pour offrir au wolof et à l'Afrique les moyens de res(t)ituer les voix perdues au large. « Dire tel quel » ou «tell it like it is ${ }^{18}$, selon les termes du poète-traducteur Langston Hughes (1902-1967), pourrait être le credo de toute traduction. $\mathrm{Y}$ arriver ou pas est une autre question. Il y a dans cette visée du « tel quel » que met en œuvre l'anthologie de D. Ndiaye une conviction à propos d'un territoire $(\mathrm{du})$ commun et d'une cicatrisation toujours possible de la plaie laissée par l’Histoire coloniale.

Ainsi, notre traducteur africain permute-t-il les poèmes diasporiques choisis selon l'axe géo-traducto-logique, reconnaissant la démarcation que cela implique, et commute l'élan africain distinctif qui les traverse. Si ces textes ont en effet distingué l'Afrique en la commémorant : "My song / From the dark lips / Of Africa ", chante Langston Hughes dans "Me and My Song» (Hughes, 1994, pp. 296-297); ils se sont également distingués d'elle du fait de leurs territoires, de leurs langues d'énonciation et de l'identité de leur énonciateur : "So long / So far away / Is Africa ", semble déchanter le même poète dans "Afro-American Fragment » (p. 128). Ces deux extraits - que le poète traducteur wolof rend par «Sama wóy wile / Sañcoo ci tuñ wu ñuul / Ca Afrig » et "Yagg lóol / Sori lóol / Moodi Afrig » - situent le lieu de l'articulation et l'articulation du lieu. Le chant sur la rive américaine peut être « saturé » (Henderson, 1973) d’Afrique, lui devoir sa stature/texture, s'arrimer à l'organe double (lips) - origine double? - qui protège la langue de la coupure, mais son rythme n'en est pas moins structuré par le sujet déplacé : c'est son chant à lui, et il est éloigné de la terre d'Afrique. Deux termes importent dans l'enjeu, la mise en jeu, traductologique que nous cherchons à définir : " song » et «long », le chant et le temps, la langue et la distance, ce qui est dit et ce qui est vécu. Le passage de la langue anglaise (dans laquelle la rime

18 Expression qui qualifie pour beaucoup l'œuvre du poète afro-américain Langston Hughes (1902-1967), dont quelques-uns des poèmes ont été sélectionnés par $\mathrm{D}$. Ndiaye pour son anthologie Le retour du pigeon voyageur. 
s'imprime parfaitement à la suite d'une différence consonantique minimale) au wolof s'effectue avec perte du trait linguistique susmentionné. Remplacé par un attrait, replacé dans un attirail, temporel : les deux mots trouvés en wolof (wóy et yagg) se retrouvent dans le giron du temps, dans son flux. En effet, le mot wóy traduit song (chant ou chanson qui ne se font pas sans son, ton et temps), mais recèle dans son potentiel sémantique un autre sens quand il devient verbe, sous l'impulsion de la conjugaison des temps : quand il perd son accent, woy signifie coaguler, figer, cailler ou encore solidifier. Il y a dans la restitution/resituation en wolof un passage du sens et de mémoire, comme nous l'avons déjà dit. Le chant du pigeon voyageur traduit est celui qui a duré, résisté, qui transporte une permanence même quand il porte sa différence. N'oublions pas, et nous ne nous en servons qu'à titre d'exemple contradictoire concordant, à propos du second sens de woy, l'Amérique elle-même, dans sa posture raciste, a longtemps figé le sang noir (africain) de l'Afro-américain avec sa politique du «one-drop rule ». Il suffisait " d'une goutte de sang noir ». Encore que l'usage du terme de " Noir » puisse nous entraîner dans d'autres considérations. Limitons-nous donc à la dialectique temps et chant, abordée ci-dessus, qu'on peut prolonger par un autre poème de Langston Hughes, ne se rapportant pas forcément à sa veine africaniste mais au titre fort à propos, « So Long » (Hughes, 1994, p. 413), que nous donnons avec sa tentative de traduction en wolof, « Yagg lóol »:

\author{
So long \\ Yagg lóol \\ is in the song \\ nekk ci biir wóy wi \\ and it's in the way you're gone \\ nekkit ci yóon wi nga wëyee \\ but it's like a foreign language \\ teewul mu melni lakkub gan \\ in my mind \\ ci sama xél \\ and maybe was I blind \\ amaana da maa gumboon \\ I could not see \\ ba mënuma woona giss \\ and would not know \\ te duma woonit xam \\ you're gone so long \\ ne wëy nga ci lu yagg lóol \\ so long \\ yagg lóol
}


Le temps est dans le chant. L'on aura perçu le caractère phonologique de cette approche qui s'accorde aux possibilités instrumentales de la langue, en dépit de l'écart diachronique. Il s'agit de faire le jeu de l'échange au change (Werner, 1994), de distinguer, dans le désir même de replacer des voix de descendants d'Africains du lointain en Afrique, en retour, avec une langue africaine, l'innovation proprement afro-américaine (Nielsen, 2004). Werner et Nielsen focalisent sur la musique, sur l'entrelacs musique/écriture, sur l'écriture qui se fait musique et vice versa, comme marqueur singulier de la création afro-américaine. D’aucuns ne voudront y voir que prosodie, rythme et mélodie inhérentes à la poésie alors qu'il s'agit de quelque chose de plus inaliénable : le lien viscéral de la poésie d'un Langston Hughes, d'un Sterling Brown, d'un Amiri Baraka ou d'un Yusef Komunyakaa au blues et au jazz.

Dans ce rapport étroit et même intime entre poètes afro-américains et formes musicales (re)crées hors d'Afrique (Integral Music, pour emprunter le titre de Aldon Nielsen), le poète traducteur wolof (voire africain tout simplement) se trouve défié dans son désir même de restitution. S'il y a d'une part un a priori culturel essentialiste se traduisant par le retracement du blues, jazz, voire du hip hop, aux sources africaines: I have been a singer : / All the way from Africa to Georgia / I carried my sorrow songs, écrit L. Hughes (p. 24). Il est indéniable, d'autre part, que dans ces formes musicales, il y a un langage spécifique qui échappe à l'Afrique (c'est-à-dire totalement coupé de/à l'origine) : celui de l'expérience unique vécue lors de la traversée et suite à celle-ci : "I made ragtime », dit le vers qui suit ceux cités supra). Ce second « je » est différent/différé, car déjà déplacé, c’est celui qui a fait et non pas celui qui a transbordé. Symétriquement, une traduction à visée phonologique dans l'entreprise traductologique qu'est Le retour $\mathrm{du}$ pigeon voyageur, signifierait ceci : dans le geste même du traduire vers une langue africaine de textes poétiques identifiés comme entretenant un lien évident ou latent à l'Afrique, le traducteur se confronte à la réticence intrinsèque à ces formes; autrement dit, leur intégrité en tant que formes en mouvement; ce qui doit le pousser, à son tour, à puiser dans ce retour des possibilités d'innovation pour sa propre langue ou d'épuiser le potentiel créatif de cette dernière.

Le désir clairement affirmé par $\mathrm{D}$. Ndiaye de restituer à l'Afrique la voix de ses descendants d'outre-Atlantique, de rétablir le lien, la communion, la communication, trouve aussi sa pertinence dans son horizon politique et ce, jusqu'à risquer la faillibilité dans la quête du 
sens linguistique en acceptant, d'emblée, l'échec d'une intelligibilité linguistique à la faveur d'une itérabilité historique : «[...] a notion of translation that, from the very start, assumes the ultimate failure of linguistic intelligibility while insisting on the historical iterability [...]" (Scott, 2005, p. 56) ${ }^{19}$. Scott identifie l'effet de l'Afrique sur la traduction, et sur sa propre traduction ajouterions-nous, à partir de sa position historique : "[...] "Africa," operating as the figure of a prelinguistic iterability, is the positive condition of possibility for acts of translation [...] » (Scott, 2005, p. 56). La position historique du traducteur africain d'aujourd'hui doit le pousser à opérer un acte créatif dans la reprise des créations qui du lointain ont traduit l'Afrique. Ainsi, il réitère l'implantation du temps dans la langue en replaçant les textes ciblés dans un temps et espace africains nouveaux.

\section{Durham, Paris, Université de l’Alberta}

\section{Annexe}

“The Negro Speaks of Rivers” (Langston Hughes, 1921).

I've known rivers:

I've known rivers ancient as the world and older than the Flow of human blood in human veins.

My soul has grown deep like the rivers.

I bathed in the Euphrates when dawns were young. I built my hut near the Congo and it lulled me to sleep. I looked upon the Nile and raised the pyramids above it. I heard the singing of the Mississippi when Abe Lincoln went down to New Orleans, and I've seen its muddy bosom turn all golden in the sunset.

I've known rivers :

Ancient, dusky rivers.

My soul has grown deep like the rivers.

${ }^{19}$ Dans sa lecture d'un poème de L. Hughes intitulé «Letter from Spain » (Hughes, 1994, pp. 201-202). 
“Nit ku ñuul a ngi wax ci dex yi”

Xam naa ay dex :

Xam naa ay dex yu màgget na àdduna te gën fee yàgg

Deret jiy daw ci sidditi doom aadama

Samag ruu dellu nag xóot ni dex yi

Sangu naa ci Ëfraat ba ndoortelu àdduna

nekkee cig ndaw

Tabax naa sama néegu ñax ci wetu Kongo gi ma yeetal

Xool naa Niil dellu tabax ca kow bàmmeeli buur ya

Dégg naa woyu Misisipi ba Abaraam Linkoln wàcce

Nuwel Orleyaan, gis naa fa ban bu xóot buy wal

soppiku wurus ca marax

Xam naa ay dex

Dex yu màgget te nëx.

Samag ruu dellu nag xóot ni dex yi

Traduit par Daouda Ndiaye. 


\section{Références}

BANDIA, Paul (2001). « Le concept bermanien de l' « Étranger » dans le prisme de la traduction postcoloniale ». TTR, XIV, 2, pp. 123-139.

BERMAN, Antoine (1986). « L'essence platonicienne de la traduction ». Revue d'esthétique, $\mathrm{n}^{\circ} 12$, 1986, pp. 63-73.

DALBY, David (1970). " Reflections on the classification of African languages with special reference to the work of SIGISMUND, Wilhelm Koelle and Malcolm GUTHRIE ». African Language Studies, ${ }^{\circ}{ }^{11}$, pp. 1-14.

DERRIDA, Jacques (1996). Le monolinguisme de l'autre ou la prothèse d'origine. Paris, Éditions Galilée.

DIOP, Cheikh, A. (1954). Nations nègres et culture. Paris, Présence Africaine.

GILROY, Paul (1992). The Black Atlantic: Modernity and Double Consciousness. Cambridge, Harvard UP.

GYASI, Kawaku, A. (1999). «Writing as Translation: African Literature and the Challenges of Translation ». Research in African Literature, 30, 2, pp. 75-87.

HEINE, Bernd et Derek NURSE (2000). African Languages. An Introduction. Cambridge, Cambridge University Press.

HENDERSON, Stephen (1973). Understanding the New Black Poetry: Black Speech and Black Music as Poetic References. New York, William Morrow \& Comp., Inc.

HUGHES, Langston (1994). The Collected Poems of Langston Hughes. Arnold Rampersad, Editor and David Roessel, Associate Editor, New York, Vintage Classics.

KANE, Ousmane (2003). Intellectuels non europhones. CODESRIA, url: http://www.codesria.org (consulté le 08/12/05).

MAZRUI, Alamin M. et Ibrahim N. SHARIFF (1994). The Swahili. Idiom and Identity of an African People. Trenton, Africa World Press. 
NAMA, Atangana, Charles (1993). «Historical, Theoretical and Terminological Perspectives of Translation in Africa ». Meta, 38, 3, pp. 414-425.

NDIAYE, Daouda (à paraître). Le retour du pigeon voyageur.

- (2003). Gàddaay Gi (recueil de poèmes en langue wolof). Paris, L'Harmattan.

- (1999). Keppaarug guy gi (recueil de poèmes en langue wolof). Paris, L'Harmattan.

NIELSEN, Aldon Lynn (2004). Integral Music: Languages of African American Innovation. Tuscaloosa, Alabama, University of Alabama Press.

PÉTRÉ-GRENOUILLEAU, Olivier (2004). Les traites négrières : essai d'histoire globale. Paris, Gallimard.

RAO, Sathya (2006a). «Le point de vue de la théorie unifiée de l'Afrique et son envolée dans le champ de la traduction ». In Simon Battestin (dir.). De l'écrit africain à l'oral. Le phénomène graphique africain. Paris, L’Harmattan, pp. 133-146.

- (2005). "Comment la philosophie doit-elle penser l'Autre de couleur?». Éthiopiques, 74, ${ }^{\mathrm{er}}$ semestre 2005, pp. 145-157.

- (2004, publié en 2006b). "Quelques considérations éthiques sur l'invisibilité du traducteur ou les vertus du silence en traduction ». TTR, XVII, 2, pp. 13-25.

ROBINSON, David (2000). Paths of Accommodation. Ohio, Oxford Ohio University Press.

SCOTT, Williams (2005). « Motivos of Translation: Nicolàs Guillén and Langston Hughes ». CR: The New Centennial Review 5, 2, pp. 35-71.

WERNER, Craig Hansen (1994). Playing the Changes: From Afro-Modernism to the Jazz Impulse. Urbana and Chicago, University of Illinois Press. 
WRIGHT, Richard (1941). 12 Million Black Voices, first published by Viking Press in 1941; reprinted in Wright, Ellen et Michel Fabre (1997). Richard Wright Reader. New York, Da Capo Press, pp. 144-241.

RÉSUMÉ : Traduire pour l'Afrique. Une approche géo-traducto-logique - Ce texte entend s'engager en faveur d'un traduire africain. Pour ce faire, il part du projet de traduction, initié par Daouda Ndiaye, de textes de la diaspora afro-américaine/européenne en langue wolof pour en interroger le geste même : que signifie le fait de restituer à l'Afrique l'héritage de ses fils perdus, ce que le traducteur compte accomplir à travers ses traductions? Est-il possible, par ces traductions en une langue africaine, ici le wolof, de rendre compte de ce qui a été perdu ou retenu durant le terrible voyage, mais aussi de ce qui a été (re)créé sur les nouveaux territoires (la nouveauté des créolisations)?

ABSTRACT: Translating for Africa: a Geo-translato-logical Perspective - This article actively supports the idea of an African translation. Taking as its point of departure the project of translation of Afro-American/European texts back into wolof initiated by Daouda Ndiaye, this article questions the meaning of such a journey: what does bringing back the legacy of Africa's abducted sons, one of the translator's aims, really mean? Is it possible, through these translations into an African language, in the case at hand wolof, to express what was lost or retained during the terrible journey, but also what was (re)created in the new territories (the novelty of creolisations)?

Mots-clés : Afrique, wolof, Middle Passage, traversée, traduire.

Keywords: Africa, Wolof, Middle Passage, crossing, translation.

Serigne Kandji : 18 rue des Meuniers, 75012 Paris, France

Courriel: serigne.kandji@wanadoo.fr

Daouda Ndiaye : 1 rue des Ecoles, 94000 Alfortville, France

Courriel: daouda.ndiaye@gmail.com

Sathya Rao: Département de Langues Modernes et d'Études Culturelles, Université de l'Alberta, 217-B Arts Building

Edmonton, Alberta, Canada T6G 2E6

Courriel: sathya_rao6@hotmail.com/srao@ualberta.ca 